Pacific Journal of Mathematics

ON LOCAL UNIFORM MEAN CONVERGENCE FOR MARKOV 


\title{
ON LOCAL UNIFORM MEAN CONVERGENCE FOR MARKOV OPERATORS
}

\section{Robert Sine}

\begin{abstract}
The center of a stable Markov operator on $C(X)$ is studied to obtain necessary and sufficient conditions for uniform convergence of the Cesaro means of the iterates on the center of the process. The relation between these results and previous local convergence theorems is also examined.
\end{abstract}

We denote by $C(X)$ the $B$-space of all real valued continuous functions on a compact Hausdorff space. The $w^{*}$-compact convex set of all Baire Probabilities on $X$ is denoted by $\mathscr{P}(X)$. A (stable) Markov operator $\mathrm{T}$ is a bounded linear operator on $C(X)$ which is positive and which satisfies $T 1=1$. For each $x$ in $X$ and each Baire set $E$ in $X$ we should point out that $T^{*} \delta(x) E$ can be interpreted as the probability of hitting the set $E$ at time $n=1$ having started at the point $x$ at time $n=0$. However we will make no appeal to probabilitistic interpretations or methods here. This note continues the theme that much of the asymptotic behavior of $T$ is intimately connected with the Banach space geometry of the invariant structures of $T$. We will denote the invariant function manifold for $T$ in $C(X)$ by $M=M(T)=\{f: T f=f\}$ and denote the compact convex set of invariant probabilities in $\mathscr{P}(X)$ by $\mathscr{K}(T)=\left\{\mu: T^{*} \mu=\mu\right\}$.

In $L_{2}$ ergodic theory the strong operator convergence of the Cesaro means of the iterates of a contraction is free ( $\mathrm{v}$. Neumann's ergodic theorem). For Markov operators on $C(X)$ this convergence is not free or even true. Thus we have the following definition; $T$ is uniformly mean stable (u.m. s.) on $C(X)$ if

$$
A_{n}(T) f=1 /(n+1)\left(l+T+\cdots+T^{n}\right) f
$$

is uniformly convergent for all $f$ in $C(X)$. This condition has been studied by Jamison [4], Lloyd [5], Rosenblatt [8] and Sine [9]. We will make strong use of the following separation property from [9, p. 161]. A Markov operator $T$ is u.m.s. on $C(X)$ iff $M(T)$ separates $\mathscr{K}(T)$. We will say a closed set $D$ is invariant if $T^{*} \delta(x) D=1$ for all $x$ in $D$.

Consider the following very simple example. We take the unit interval $[0,1]$ as $X$ and define $T f(x)=f\left(x^{2}\right)$. Then $T^{n} f$ will converge pointwise so $A_{n}(T) f$ will converge pointwise to the same limit. However this limit function will be continuous iff $f(0)=f(1)$ so $T$ 
is not u.m.s. But the doubleton set $M=\{0,1\}$ is invariant and the restricted operator $\left.T\right|_{M}$ is u.m.s. on $M$. (Indeed $\left.T\right|_{M}$ is the identity operator.) It is this sort of local u.m.s. we will characterize.

2. We define the center of a Markov operator $T$ by

$$
M=\text { closure } U\{\operatorname{supp}(\lambda): \lambda \text { in } \mathscr{K}(T)\} \text {. }
$$

This definition is suggested by the center of attraction in dynamic systems (see Nemytskii and Stepanov [6, p, 367]). We can obtain $M$ in another way. Let $\boldsymbol{J}$ be defined by

$$
J=\left\{f: A_{n}(T)|f| \rightarrow 0 \text { [pointwise] }\right\} .
$$

Then firstly the convergence is uniform by Dunford's mean ergodic theorem [2,p. 661]. Secondly it is easy to show that $J$ is a norm closed ideal in $C(X)$ which is $T$ invariant as a set of functions. Thus the zero set of the ideal is a closed $T$ invariant set in $X$. Finally it is easy to show that this zero set is the center $M$. Thus it can be shown that if $f$ vanishes on $M$ we have $A_{n}(T) f$ uniformly convergent to zero. (See (See [11] for details)

The definition of $M$ is unchanged if the probabilities, $\lambda$, are only taken from the extreme points of $\mathscr{K}(T)$. This is an easy consequence of the Krein-Milman theorem; we leave the details to the reader. It is perhaps more surprising that in the case that $X$ is metrizable the definition is unchanged with the omission of the closure operation.

THEOREM 1. Let $K$ be a sequentially closed convex subset of $\mathscr{P}(T)$ with $X$ compact Hausdorff. Then $M=U\{\operatorname{supp}(\lambda): \lambda \varepsilon K\}$ is sequentially closed.

Proof. Suppose $\left\{x_{n}\right\}$ is a sequence of points in $M$ and $x_{n} \rightarrow y$. Then there exists a sequence $\left\{\lambda_{n}\right\}$ in $K$ with $x_{n}$ in $\operatorname{supp}\left(\lambda_{n}\right)$. If we set $\mu=\Sigma\left(2^{-n}\right) \lambda_{n}$ then $\mu$ is in $K$. If $W$ is any neighborhood of $y$ then $\left\{x_{n}\right\}$ is ultimately in $W$ so $\lambda_{n}(W)>0$ ultimately. Hence $\mu(W)>0$ so $y$ is in $\operatorname{supp}(\mu)$. This finishes the argument.

We will consider properties which are too weak or too strong before our main result. We introduced in [9] the concept of a topological ergodic decomposition for $T$. This property was shown to imply u.m.s. on a superset of the center but it is much too strong a condition. It required (among other things) a sufficiently rich family of invariant functions to separate the extreme points of $\mathscr{K}(T)$. But our example above has only constants for invariant functions while it is u.m.s. on its center. 
We next look at a condition which is too weak. We will say $T$ is scattered if each pair of distinct extreme invariant probabilities in $\mathscr{K}(T)$ have disjoint supports.

THEOREM 2. If $D$ is minimal invariant set for a scattered Markov operator $T$ the $\left.T\right|_{D}$ is u.m.s.

Proof It is clear that $D$ supports exactly one extreme invariant probability. It follows from Krein-Milman that $\left.T\right|_{D}$ is uniquely ergodic and it is well known that uniquely ergodic implies u.m.s. (see Oxtoby [7, p. 124]).

REMARK. It follows from the above theorem that $A_{n}(T) f$ converges pointwise on the union of the minimal invariant sets if $T$ is scattered. Note that each extreme invariant probability is supported on a minimal set if $T$ is scattered. If the union of the minimal sets is closed and if the minimal invariant sets form an upper semi-continuous decomposition as well then the scattered Markov operator is u.m.s. on its center. But neither of these topological conditions for a scattered Markov operator need hold. First an example of a scattered operator with union of the minimal sets not closed. We take $X=[0,1] \times[0,1]$ and define the operator by

$$
T f(x, y)=y f(0, y)+(1-y) \int f(s, y) d s .
$$

It is straightforward to show that the minimal sets are each uniquely ergodic and consist of the horizontal fibers $I(y)=\{(x, y): 0 \leqq x \leqq 1\}$ for $0 \leqq y<1$ together with the singleton point $(0,1)$.

For an example with the union closed but not an upper semicontinuous decomposition take on the same space $X$

$$
T f(x, y)=y f(x, y)+(1-y) \int f(s, y) d s .
$$

The minimal invariant sets consist of the fibers $I(y)$ with $0 \leqq y<1$ again together with the singleton points $\{(x, 1)\}$ for $0 \leqq x \leqq 1$. Finally we remark that these topological conditions are too strong as a u.m.s. operator need not have the union of its minimal invariant sets closed.

We will say $T$ is continuously scattered if there is a family of continuous functions each constant on the support of each extreme invariant probability and sufficient to separate the extreme invariant probabilities.

THEOREM 3. A Markov operator is continuously scattered iff it is u.m.s. on its center. 
Proof. If $T$ is u.m.s. on its center then the invariant functions for $\left.T\right|_{M}$ when extended to all of $X$ in any continuous way satisfy the requirements. Conversely suppose there is such a family of functions. We have $T f=f$ on each minimal invariant set and thus on the union. By continuity $T f=f$ on $M$. But in the same way we have $T g=g$ on $M$ for each function in the norm closed algebra $\mathscr{A}$ generated by the separating family. Let $Y$ be the quotient space $M / \mathscr{A}$. We can drop $T$ to a Markov operator on $C(Y)$; it is, in fact, the identity operator on $C(Y)$. It follows that for each $y$ in $Y$ that the pre-image of $y$ under the quotient map is a closed invariant set of $M$ which supports exactly one invariant probability. Thus $T$ is u.m.s. (by unique ergodicity) on that pre-image. Now let $\lambda_{1}$ and $\lambda_{2}$ be any invariant probabilities. Let $f$ be any function with $\left(f, \lambda_{1}\right) \neq\left(f, \lambda_{2}\right)$. Now $\bar{f}=\lim A_{n} f$ exists as a pointwise limit on $M$. This function separates $\lambda_{1}$ and $\lambda_{2}$ and it can be regarded as a Baire function on $Y$. But then there must be a continuous function on $Y$ separating $\lambda_{1}$ and $\lambda_{2}$. Finally any function of $C(Y)$ is $\left.T\right|_{M}$ invariant when regarded as a function on $M$. Thus the invariant functions of $\left.T\right|_{M}$ separate $\mathscr{K}(T)$ so $T$ is u.m.s. on its center.

The following condition was given by Attala [1]. Let $\mathscr{L}_{0}$ be the norm closed subspace of $C(X)$ defined by

$$
\mathscr{L}_{0}=\left\{f: A_{n}(T) f \rightarrow 0 \text { [uniformly] }\right\} .
$$

Suppose there is a Markov projection $P$ with the null space of $P$ equal to $\mathscr{L}_{0}$. Then $T$ is u.m.s. on its center. We will give a converse to this result in the metric case thus further justifying the hypothesis. We will also give an apparent strengthening of the forward theorem in such a way that it is clear that for $T$ to be u.m.s. on $M$ depends only on $\mathscr{K}(T)$ and its orientation in $\mathscr{P}(X)$.

THEOREM 4. Let $T$ be a Markov operator on $X$ with center $M$. Suppose there is a u.m.s. Markov operator $S$ on $C(X)$ with $K(S)=K(T)$. Then $T$ is u.m.s. on $M$.

Proof. First we note that if $R$ is any stable Markov operator and $\lambda$ is in $\mathscr{K}(R)$ then $\operatorname{supp}(\lambda)$ is an $R$-invariant set (see $[9, \mathrm{p} .156])$. It follows that $M$ is both $T$ and $S$ invariant and we can restrict the processes to $M$. If $\lambda$ is an extreme point of $\mathscr{K}=\mathscr{K}(S)=\mathscr{K}(T)$ then $D=\operatorname{supp}(\lambda)$ is both $S$ and $T$ invariant. Moreover since $S$ is uniquely ergodic on $D$ so is $T$. Now each $S$ invariant function is constant on $D$. Let $f$ be an $S$ invariant function. Then $T f=f$ on the support of each extreme since $f$ is constant on each such set. Then by continuity $T f=f$ on $M$ since as pointed out before $M$ is the closure of the union of the supports of the extremes. Now each $S$ invariant function is $T$ 
invariant and each $T$ invariant probability is $S$ invariant. Since $S$ is u.m.s. the $S$ invariant functions separate the extremes of $\mathscr{K}(S)=$ $\mathscr{K}$. Also the $T$ invariant functions separate the extremes of $\mathscr{K}=$ $\mathscr{K}(T)$. We conclude $T$ is u.m.s. on $M$.

REMARK. If $T$ is a Markov operator on $C(X)$ and $T$ is u.m.s. on $M$ then clearly there is a u.m.s. Markov operator $S$ on $C(X)$ with $\mathscr{K}(S)=\mathscr{K}\left(\left.T\right|_{M}\right)$. For we just take $S=\left.T\right|_{M}$. Now in the above theorem we only need $S$ u.m.s. on $M$ and $S$ need not even be defined on all of $X$. To that extent the conditions of Theorem 4 (and of Attala's result as well) are necessary and sufficient. But if we ask that $S$ be globally defined we are able to obtain a full converse only in the metric case.

THEOREM 5. Let $T$ be a Markov operator on $C(X)$ where $X$ is compact metric. Then $T$ is u.m.s. on its center $M$ iff there is a u.m.s. Markov operator $S$ on $C(X)$ with $\mathscr{K}(S)=\mathscr{K}(T)$.

We will have need of the following.

Lemma. (Borsuk-Tietze). Let $X$ be a compact metric space and $D$ a closed nonempty subset. Then there is a Markov projection $P$ with $\mathscr{K}(P)=\mathscr{P}(D)$.

Borsuk's linear Tietze extension result has far greater generality than this lemma. For the lemma as stated a geometric proof is available based on the strictly convex metrics of Bonsall and Hervé for the $w^{*}$ topology [10].

Proof of Theorem 5. There is a Markov projection $R$ with $\mathscr{K}(R)=\mathscr{P}(M)$ by the lemma. Let $P$ be the projection on $C(X)$ defined as the limit of $A_{n}\left(\left.T\right|_{M}\right)$. For each $x$ in $X$ we have $P^{*} R^{*} \delta(x)$ defined as a point in $\mathscr{K}(T)$. If $\lambda$ is in $\mathscr{K}(T)$ then $P^{*} R^{*} \lambda=\lambda$. Thus $S=R P$ is a Markov projection with $\mathscr{K}(S)=\mathscr{K}(T)$ and satisfies the requirements. For $f$ in $C(X)$ we let $P$ act on the restriction to $M$. Let $g$ be any extension of $P f$ to all of $X$. Then $R P f=R g$ is independent of that extension.

\section{REFERENCES}

1. R. Atalla, On the mean convergence of Markov operators, to appear in Proc. Edinburgh Mathematical Society.

2. N. Dunford and J. Schwartz, Linear Operators, part I, Interscience, New York, 1958.

3. B. Jamison, Ergodic decompositions inducted by certain Markov operators, Trans. Amer. Math. Soc., 117 (1965), 451-468. 
4. S. Lloyd, On certain projections in spaces of continuous functions, Pacific J. Math., 13 (1963), 171-175.

5. V. Nemytskii and V. Stepanov, Qualitative Theory of Differential Equations, Princeton University Press, Princeton, 1960.

6. J. Oxtoby, Ergodic Sets, Bull. Amer. Math. Soc., 58 (1952), 116-136.

7. M. Rosenblatt, Equicontinuous Markov operators, Teor. Veronjatnost. i Primenen. 9 (1964), 205-222.

8. R. Sine, Geometric theory of a single Markov operator, Pacific J. Math., 27 (1968), 155-166.

9. - The Borsuk-Tietze linear extension with a side condition, to appear, J. Approx. Theory. 10. Sample path convergence of stable Markov processes II, to appear in Indiana J. Math.

Received June 26, 1974. Partially supported by National Science Foundation GU-3171.

State University of New York at Albany

Current address: University of Rhode Island 


\section{PACIFIC JOURNAL OF MATHEMATICS}

\section{EDITORS}

RICHARD ARENS (Managing Editor)

University of California

Los Angeles, California 90024

\author{
R. A. Beaumont \\ University of Washington \\ Seattle, Washington 98105
}

\section{J. DugundII}

Department of Mathematics

University of Southern California

Los Angeles, California 90007

D. Gilbarg and J. Milgram

Stanford University

Stanford, California 94305

\section{ASSOCIATE EDITORS}
E. F. BECKENBACH
B. H. NeumanN
F. WoLF
K. YoshidA

\section{SUPPORTING INSTITUTIONS}

\author{
UNIVERSITY OF BRITISH COLUMBIA \\ CALIFORNIA INSTITUTE OF TECHNOLOGY \\ UNIVERSITY OF CALIFORNIA \\ MONTANA STATE UNIVERSITY \\ UNIVERSITY OF NEVADA \\ NEW MEXICO STATE UNIVERSITY \\ OREGON STATE UNIVERSITY \\ UNIVERSITY OF OREGON \\ OSAKA UNIVERSITY
}

\author{
UNIVERSITY OF SOUTHERN CALIFORNIA \\ STANFORD UNIVERSITY \\ UNIVERSITY OF TOKYO \\ UNIVERSITY OF UTAH \\ WASHINGTON STATE UNIVERSITY \\ UNIVERSITY OF WASHINGTON \\ AMERICAN MATHEMATICAL SOCIETY
}

The Supporting Institutions listed above contribute to the cost of publication of this Journal, but they are not owners or publishers and have no responsibility for its contents or policies.

Mathematical papers intended for publication in the Pacific Journal of Mathematics should be in typed form or offset-reproduced (not dittoed), double spaced with large margins. Underline Greek letters in red, German in green, and script in blue. The first paragraph or two must be capable of being used separately as a synopsis of the entire paper. Items of the bibliography should not be cited there unless absolutely necessary, in which case they must be identified by author and Journal, rather than by item number. Manuscripts, in duplicate, may be sent to any one of the four editors. Please classify according to the scheme of Math. Reviews, Index to Vol. 39. All other communications should be addressed to the managing editor, or Elaine Barth, University of California, Los Angeles, California, 90024.

100 reprints are provided free for each article, only if page charges have been substantially paid. Additional copies may be obtained at cost in multiples of 50.

The Pacific Journal of Mathematics is issued monthly as of January 1966. Regular subscription rate: $\$ 72.00$ a year (6 Vols., 12 issues). Special rate: $\$ 36.00$ a year to individual members of supporting institutions.

Subscriptions, orders for back numbers, and changes of address should be sent to Pacific Journal of Mathematics, 103 Highland Boulevard, Berkeley, California, 94708.

PUBLISHED BY PACIFIC JOURNAL OF MATHEMATICS, A NON-PROFIT CORPORATION Printed at Jerusalem Academic Press, POB 2390, Jerusalem, Israel.

\section{Copyright (C) 1975 Pacific Journal of Mathematics All Rights Reserved}




\section{Pacific Journal of Mathematics

Vol. 60, No. $2 \quad$ October, 1975

Waleed A. Al-Salam and A. Verma, A fractional Leibniz q-formula ........... 1

Robert A. Bekes, Algebraically irreducible representations of $L_{1}(G) \ldots \ldots \ldots \ldots 11$

Thomas Theodore Bowman, Construction functors for topological

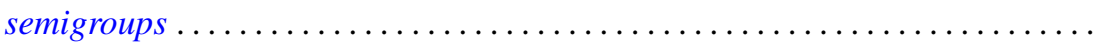

Stephen LaVern Campbell, Operator-valued inner functions analytic on the

closed disc. II .........................................

Leonard Eliezer Dor and Edward Wilfred Odell, Jr., Monotone bases in $L_{p} \ldots \ldots$.

Yukiyoshi Ebihara, Mitsuhiro Nakao and Tokumori Nanbu, On the existence of

global classical solution of initial-boundary value problem for

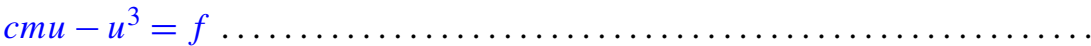

Y. Gordon, Unconditional Schauder decompositions of normed ideals of

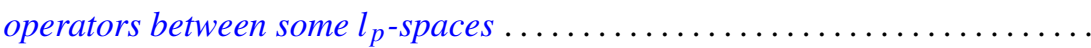

Gary Grefsrud, Oscillatory properties of solutions of certain nth order functional

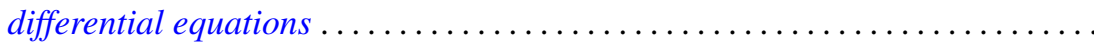

Irvin Roy Hentzel, Generalized right alternative rings ...................

Zensiro Goseki and Thomas Benny Rushing, Embeddings of shape classes of compacta in the trivial range .................................

Emil Grosswald, Brownian motion and sets of multiplicity . .

Donald LaTorre, A construction of the idempotent-separating congruences on a

bisimple orthodox semigroup .

Pjek-Hwee Lee, On subrings of rings with involution ...

Marvin David Marcus and H. Minc, On two theorems of Frobenius ...

Michael Douglas Miller, On the lattice of normal subgroups of a direct

product. .

Grattan Patrick Murphy, A metric basis characterization of Euclidean space

Roy Martin Rakestraw, A representation theorem for real convex functions ....

Louis Jackson Ratliff, Jr., On Rees localities and $H_{i}$-local rings ...

Simeon Reich, Fixed point iterations of nonexpansive mapping . .

Domenico Rosa, $B$-complete and $B_{r}$-complete topological algebras ...

Walter Roth, Uniform approximation by elements of a cone of real-valued

functions ....

Helmut R. Salzmann, Homogene kompakte projektive Ebenen

Jerrold Norman Siegel, On a space between $B H$ and $B_{\infty} \ldots$

235

Robert C. Sine, On local uniform mean convergence for Markov operators

James D. Stafney, Set approximation by lemniscates and the spectrum of an

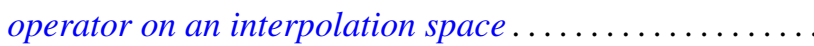

Árpád Száz, Convolution multipliers and distributions .......

Kalathoor Varadarajan, Span and stably trivial bundles ..........

Robert Breckenridge Warfield, Jr., Countably generated modules over

commutative Artinian rings....................... 